\title{
CLASSIFICATION OF SEMANTIC RELATIONS OF THE TWO-STEP EPITHET'S COMPONENTS IN PARADIGMATIC AND SYNTAGMATIC ASPECTS
}

\author{
Olga L. Lyubovskaya \\ Saint Petersburg State University, Saint Petersburg, Russia
}

\begin{abstract}
The paper reveals structural and semantic peculiarities of the two-step epithet. Within the frames of studying the linguistic nature of the two-step epithet, we give its definition, distinguish four morphologicalsyntactic models, reveal the affective and expressive potential of these lexical units. It has also been stated that standard semantic classifications of epithets prove to be inconsistent from the viewpoint of their application in the analysis of the two-step epithets due to their dual nature. Taking its paradigmatic and syntagmatic characteristics into consideration, our classification of two-step epithet describes semantic relations in its structure in complete and consistent way. Two-step epithets have been subdivided into metaphoric and metonymic on the basis of paradigmatic criterion. From the viewpoint of syntagmatics, we have distinguished specifying, synonymic, and oxymoron epithets, in which the relations between their components are those of mutual complementarity, identity and contrast. Apart from these types, a number of rarely used two-step epithets can be distinguished. The semantic relations between their components are represented as tautology, inequality, quality, quantity, and irony. We mark the flexibility of boundaries between the types of semantic relations in the structure of the two-step epithet.

The research material is represented with 540 two-step epithets sampled from original English fiction of the $19^{\text {th }}-20^{\text {th }}$ centuries.

Key words: two-step epithet, semantic relations, paradigmatic aspect, syntagmatic aspect, English language.

Citation. Lyubovskaya O.L. Classification of Semantic Relations of the Two-Step Epithet's Components in Paradigmatic and Syntagmatic Aspects. Vestnik Volgogradskogo gosudarstvennogo universiteta. Seriya 2, Yazykoznanie [Science Journal of Volgograd State University. Linguistics], 2018, vol. 17, no. 1, pp. 115-123. (in Russian). DOI: https://doi.org/10.15688/jvolsu2.2018.1.12
\end{abstract}

\section{КЛАССИФИКАЦИЯ СЕМАНТИЧЕСКИХ ОТНОШЕНИЙ КОМПОНЕНТОВ ДВУХСТУПЕНЧАТОГО ЭПИТЕТА В ПАРАДИГМАТИЧЕСКОМ И СИНТАГМАТИЧЕСКОМ АСПЕКТАХ}

\footnotetext{
Ольга Леонидовна Любовская

Санкт-Петербургский государственный университет, г. Санкт-Петербург, Россия

Аннотация. Статья раскрывает структурные и семантические особенности двухступенчатого эпитета. В рамках исследования лингвистической природы эпитета данного типа предложено авторское определение рассматриваемого феномена, выделены четыре морфолого-синтаксические модели двухступенчатых эпитетов, раскрыт эмоционально-оценочный и экспрессивный потенциал этих образований. Показано, что традиционные семантические классификации эпитетов не могут быть применены при анализе двухступенчатого эпитета в силу его двусторонней природы. Предложенная автором семантическая классификация двухступенчатых эпитетов, учитывающая их парадигматические и синтагматические свойства, позволяет полно и
} 
непротиворечиво описать семантические связи в их структуре. На основе парадигматического критерия двухступенчатые эпитеты подразделяются на метафорические и метонимические. На основе синтагматического критерия выделены широко представленные в текстах уточняющие, синонимические, оксюморонные эпитеты, в которых отношения между компонентами реализуются как взаимодополнительность, тождество, контраст, а также ряд мало употребительных эпитетов, в которых отношения между компонентами основаны на тавтологии, неравенстве, качестве, количестве, иронии. Отмечена гибкость границ между видами семантических связей в структуре двухступенчатого эпитета.

Материалом исследования послужили 540 двухступенчатых эпитетов, извлеченных методом сплошной выборки из произведений англоязычной художественной литературы XIX-XX веков.

Ключевые слова: двухступенчатый эпитет, семантические отношения, парадигматический аспект, синтагматический аспект, английский язык.

Цитирование. Любовская О. Л. Классификация семантических отношений компонентов двухступенчатого эпитета в парадигматическом и синтагматическом аспектах // Вестник Волгоградского государственного университета. Серия 2, Языкознание. - 2018. - Т. 17, № 1. - C. 115-123. - DOI: https://doi.org/10.15688/ jvolsu2.2018.1.12

\section{Введение}

Основной задачей данной статьи является классификация семантических отношений между компонентами двухступенчатого эпитета (далее - ДЭ). В качестве материала исследования избраны произведения англоязычной художественной литературы XIX$\mathrm{XX}$ веков. Прежде чем приступить к решению поставленной задачи, необходимо уточнить, что представляет собой двухступенчатый эпитет, в чем заключается его лингвистическая природа. ДЭ прежде не был предметом отдельного изучения. В работах отечественных и зарубежных языковедов, посвященных вопросам теории эпитета в целом, ДЭ рассматривается лишь как один из структурных типов эпитета (см., например: [Арнольд, 1999; Гальперин, 1958; Жирмунский, 1992; Corbett, 1969; Murphy, 2003]) и упоминается в некоторых грамматических классификациях эпитета. В частности, И.Р. Гальперин выделяет модель adverb + adjective, которую называет «наречием при прилагательном» (her eyebrows were boldly black, a hellish dull empty room). Данная модель характерна для художественных текстов [Гальперин, 1958, с. 140-141]. Л.А. Турсунова в своей классификации называет этот вид эпитета «эпитетом, усиленным наречием» или «эпитетом к эпитету» (dreadfully unsympathetic smile, burningly intimate whisper) [Турсунова, 1973, с. 27]. В классификации В.А. Кухаренко данный эпитет назван собственно двухступенчатым эпитетом («two-step epithet») (unnaturally mild day) [Kukharenco, 1986, с. 81]. Исследовате- ли выделяют ДЭ как отдельный структурный тип эпитета на основе его морфолого-синтаксических характеристик и рассматривают его в рамках грамматических классификаций.

Двухступенчатый эпитет представляет собой ядерное словосочетание с зависимыми компонентами: наречием образа действия и прилагательным или причастием, имеющими фиксированное положение относительно друг друга. ДЭ формируются в соответствии с четырьмя основными моделями:

- adverb + adjective + noun / pronoun: painfully clear head (Kipling-3, c. 199);

- adverb + participle + noun / pronoun: stupidly frightened eyes (Conrad);

- noun / pronoun + adverb + adjective: eyes inquisitively sharp (Dickens-2, p. 416);

- noun / pronoun + adverb + participle: something savagely damaging (Dickens-1, p. 122).

Наличие адвербиального и атрибутивного компонентов в структуре ДЭ позволяет говорить о том, что исследуемый тип эпитета семантически и стилистически сложнее простого эпитета. В нем один или оба компонента обладают эмоционально-оценочными и экспрессивными оттенками и могут образно характеризовать определяемое слово. Например, в составе ДЭ ball... lunarly pale (Updike), wildly virginal sea (Fitzgerald), misleadingly fastidious mouth (Fowles-1, c. 47) адвербиальный и атрибутивный компоненты являются художественно-выразительными средствами с метафорическими или метонимическими характеристиками. Следовательно, ДЭ имеет тропический характер. Несмотря на то что гораздо чаще встречаются структуры, где только один из 
О.Л. Любовская. Классификация семантических отношений компонентов двухступенчатого эпитета

компонентов является экспрессивным и создает образ, например: arrogantly curled tips (Kipling-2, p. 147), smile inexpressibly sweet (Lewis), inappropriately bourgeois teeth (Fitzgerald), такие эпитеты необходимо отнести к ДЭ по структурному признаку.

Помимо грамматических и семантических особенностей, ДЭ могут характеризоваться необычным звуковым строем. Так, ДЭ darkly distrustful eyes (Dickens-2, p. 518); dreadfully dull house (James, p. 130) представляют собой высказывания, характеризующиеся аллитерацией.

Адвербиальный и атрибутивный компоненты в структуре ДЭ способствуют большей актуализации двуплановости изображения, вторичной семантической функции, семантической асимметрии компонентов, свойственной эпитету в целом, а также разнообразию семантических отношений в его структуре, что позволяет выделить ДЭ как отдельный тип эпитета.

Учитывая грамматические, семантические и стилистические характеристики эпитетов указанного типа, мы предлагаем определение двухступенчатого эпитета как языкового средства с морфолого-синтаксической структурой «наречие + прилагательное / причастие», компоненты которого имеют семантические признаки, отличающие эпитет в целом, могут обладать эмоционально-оценочными и экспрессивными коннотациями и способны дать образную характеристику определяемому объекту или явлению.

\section{Методика исследования}

Общий объем проанализированного материала составляет 540 ДЭ. Источником материала являются более 300 оригинальных художественных произведений XIX-XX веков. Отбор материала осуществлялся методом сплошной выборки и методом корпусного анализа в тех случаях, когда возникала необходимость установления частотности употребления конкретного ДЭ или сочетаемости компонента атрибутивной группы. Корпусы были проиндексированы при помощи программы $d t$ Search, имеющей гибкую систему поисковых настроек. В качестве дополнительного источника данных в исследовании использо- ван Корпус современного американского варианта английского языка (The Corpus of Contemporary American English).

Грамматическая осложненность эпитетов данного типа обусловливает необходимость анализа семантических связей компонентов ДЭ с применением подходов парадигматической и синтагматической семасиологии.

Природа семантических отношений раскрывается в работах многих отечественных и зарубежных лингвистов. В частности, А. Круз определяет семантические отношения как отношения между понятиями, точнее, между понятийными единствами, которые могут быть связаны синтагматическими, парадигматическими и производными отношениями (discrete conceptual units: paradigmatic, syntagmatic, derivational) [Cruse, 2011, p. 125]. Вопрос о специфике парадигматических и синтагматических связей между единицами языка отражен в работах отечественных и зарубежных исследователей (см., например: [Сусов, 1970; Peters, Weller, 2008]).

Традиционно выделяются два основных типа эпитетов: постоянный эпитет и перенесенный эпитет. Так, к постоянным эпитетам Б.В. Томашевский относит эпитеты тавтологические, буквально повторяющие определяемое слово (чудо чудное, тьма тьмущзая), типические (море синее, сырая земля), идеализирующие (солнце красное) [Томашевский, 2010, с. 187].

Перенесенный эпитет имеет образную составляющую. А.Н. Веселовский называет перенесенные эпитеты синкретическими, указывая на то, что они вызваны ассоциациями наших чувственных восприятий [Веселовский, 1989]. К данному типу относят эпитеты метафорические [Веселовский, 1989; Томашевский, 2010] и метонимические [Веселовский, 1989; Сандакова, 2006].

Несколько иначе семантическая классификация эпитетов представлена в работах И.Р. Гальперина: эпитеты делятся на ассоциированные и неассоциированные [Galperin, 1971, p. 153]. Ассоциированными называются эпитеты, указывающие на такие признаки предмета, которые присущи ему по природе: triumphant pleasure, malignant torture. Крайней степенью ассоциированности характеризуется тавтологический эпитет, который по- 
вторяет признак, заложенный в самом определяемом компоненте: the heartless brutality. Неассоциированным является эпитет, указывающий на признаки, не присущие предмету. Неассоциированные эпитеты ввиду широкого разнообразия семантических отношений делятся на образные, то есть такие, в основе которых лежит какой-то зрительный, звуковой, тактильный и т. п. образ, и безобразные. В группе образных эпитетов можно выделить метафорический, синестетический и звукообразный эпитет. К группе безобразных эпитетов относятся перенесенный, оксюморонный, гиперболический и антономасийный эпитет.

Иначе строится классификация семантических связей компонентов ДЭ. Типология семантических отношений в структуре ДЭ опирается на классификацию стилистических ресурсов, представленную в работе Ю.М. Скребнева, обусловлена особенностями эпитетов исследуемого типа и осуществлена в парадигматико-синтагматическом аспекте [Скребнев, 2003, c. 149-160; Skrebnev, 1994, p. 97-122, 143-167].

Парадигматика как одна из отраслей стилистики рассматривает проблемы выбора номинации, исходя из того, что язык представляет собой множество парадигм. Слова и выражения, традиционно используемые для характеристики определенного класса объектов, могут быть использованы по отношению к объектам другого класса. В связи с этим Ю.М. Скребнев видит задачу парадигматической семасиологии в том, чтобы классифицировать семантические типы переноса и логические законы, по которым эти переносы осуществляются, и выделяет фигуры качества, к которым относит метафору, метонимию и иронию, и фигуры количества, включающие гиперболу и мейозис.

В отличие от парадигматической синтагматическая семасиология связана со стилистическими функциями отношений между единицами в текстах и изучает типы линейной организации значений. В данном ракурсе Ю.М. Скребнев выделяет возможность реализации эпитетом семантических отношений тождества, неравенства, взаимодополнительности и контраста.
ДЭ в силу своей структуры является средством семантически более сложным, чем простой эпитет, поскольку, с одной стороны, его заданная морфолого-синтаксическая структура усиливает его синтагматическую природу, с другой стороны, наличие в нем адвербиального и атрибутивного компонентов позволяет реализовать больше вариантов семантических отношений, усиливая его парадигматические характеристики. Поэтому с учетом двойственной природы ДЭ его классификация осуществлена в двух ракурсах: парадигматическом и синтагматическом. Данный подход позволяет полно и непротиворечиво описать стилистические ресурсы языка.

В парадигматическом ракурсе можно выделить

- метафорический ДЭ;

- метонимический ДЭ.

В рамках синтагматической семасиологии с учетом морфолого-синтаксических характеристик компонентов в составе ДЭ выявлены следующие виды преобладающих отношений:

- отношения взаимодополнительности (уточняющий ДЭ);

- отношения тождества (синонимический ДЭ);

- отношения контраста (оксюморонный ДЭ).

В ДЭ могут быть реализованы и другие отношения.

Так, в рамках парадигматики выделены:

- отношения количества (гиперболический ДЭ);

- отношения, реализующие иронию (иронический ДЭ).

В синтагматическом аспекте можно выделить:

- отношения неравенства (антиклимакс);

- тавтологические отношения (тавтологический ДЭ);

- отношения качества (антономасийный ДЭ).

Однако ДЭ с такими видами семантической связи представлены единичными случаями и требуют дальнейшего изучения.

Установленные синтагматические связи могут наблюдаться как между атрибутивной группой и определяемым компонентом, так и внутри атрибутивной группы. 


\section{Двухступенчатый эпитет в парадигматическом аспекте}

Метафорический и метонимический виды ДЭ выделяются по признаку переноса значения по сходству (для метафорического) и смежности (для метонимического). Метафорический и метонимический ДЭ выполняют функцию актуализации, выявляя существенный признак предмета или явления.

В структуре ДЭ метафорический перенос осуществляется в рамках значения одного из компонентов атрибутивной группы. Так, в эпитетах weirdly antiquated slang (Fowles-1, c. 49), icily jealous wife (Collins) наречие icily и прилагательное antiquated становятся метафорическими предикатами, употребленными по отношению к определяемому компоненту на основе сходства. В составе ДЭ неметафорический компонент может частично эксплицировать метафору, выполнять пояснительную или конкретизирующую функцию. В метафорическом ДЭ diabolically silent laugh (Dickens-2, p. 545) единица diabolically в контексте высказывания на основе предметно-логического значения «characteristic of the Devil, or so evil as to recall the Devil» (OALD) реализует переносное значение «horrible, fearful». Таким образом, в основе лежит метафора diabolical laugh, которая широко представлена в художественной литературе. Прилагательное silent, конкретизируя наречие diabolically, усиливает выразительность эпитета.

Примеры метафорического ДЭ составляют $11 \%$ от общего объема изученного материала.

Метонимия в ДЭ реализуется на основе ассоциативной связи определяемого компонента и его свойства, выраженного наречием: arrogantly curled tips (Kipling-2, p. 147) или прилагательным: clergyman's inappropriately bourgeois teeth (Fitzgerald). В некоторых случаях метонимический перенос реализуется между определяемым компонентом и его свойствами или признаками, выраженными атрибутивными компонентами: eyelids, dispassionately bored (Fitzgerald), the nose politely inconspicuous (Fitzgerald). Чаще всего объектом метонимии являются черты внешнего облика, посредством описания ко- торых раскрываются особенности характера и поведения, эмоциональное состояние персонажа. Как показал корпусный анализ, метонимический эпитет является одним из наиболее распространенных видов ДЭ. Для многих эпитетов с рассматриваемым видом семантических отношений характерна определенная степень устойчивости в англоязычной художественной литературе. Частотность их употребления, фиксированность метафорического значения одного или двух компонентов атрибутивной группы в словарях приводит к тому, что постепенно такой эпитет приобретает стертый характер, зачастую утрачивая свою художественно-выразительную ценность. К стертым ДЭ этой группы относятся, напримep, kindly shining eyes (Maugham, c. 95), mutually vivid memory (Fitzgerald), her faintly tired beauty (Fitzgerald), face unnaturally pale (Fitzgerald).

В парадигматическом аспекте по количеству примеров преобладает метонимический ДЭ (33 \%).

\section{Двухступенчатый эпитет в синтагматическом аспекте}

Отношения взаимодополнительности реализуются в структуре ДЭ в том случае, если его компоненты уточняют или поясняют друг друга. Такие отношения реализуются между атрибутивной группой и определяемым компонентом. Наречие и прилагательное уточняют, конкретизируют, проясняют значение определяемого компонента, описывая его более точно и полно. Компоненты являются ко-референтными, то есть характеризующими один объект. Например, в составе уточняющего ДЭ disgustingly happy family (Fowles-2, с. 185) атрибутивные компоненты семантически неоднородны и реализуют разные значения. Наречие disgustingly указывает на внешний оценочный признак: «extremely unpleasant or unacceptable» (CALD), тогда как компонент happy выражает внутреннее состояние персонажей: «feeling pleased or satisfied» (MED). Тем не менее весь семантический комплекс формирует целостное представление об объекте изображения.

Отношения взаимодополнительности являются преобладающими - 30 \% от обще- 
го числа проанализированных ДЭ относятся к уточняющему типу эпитета.

Отношения тождества наблюдаются в структуре синонимического ДЭ. Они возникают в тех случаях, когда компоненты ДЭ реализуют близкие значения, имеют общие семантические признаки, выполняют функцию уточнения и в определенных контекстах способны замещать друг друга. Так, значения атрибутивных компонентов в ДЭ recklessly daring heroes (Kipling-1) пересекаются по общему признаку: risky - «doing something dangerous and not worrying about the risks and the possible results» (CALD), daring - «brave and taking risks» (CALD). В составе синонимического ДЭ отношения сходства реализуются внутри атрибутивной группы, то есть между наречием и прилагательным, и только при наличии у них общей семы.

Случаи использования полных синонимов в ДЭ, например: drowsily languid utterance (Collins), в исследуемом материале единичны.

Синонимический тип ДЭ составляет $12 \%$ от общего количества ДЭ.

Отношения контраста основаны на антонимичности компонентов ДЭ. Они реализуются в тех случаях, когда в структуре ДЭ соединяются лексические единицы, выражающие два противоположных понятия, актуализируются два противоположных значения. ДЭ с таким типом взаимодействия компонентов можно называть оксюморонным. В оксюморонном эпитете основное предметно-логическое значение атрибутивной группы в большей или меньшей степени подчиняется экспрессивности. Например, в таком ДЭ, как painfully clear head (Kipling-3, c. 199), выражение субъективного отношения автора к описываемому предмету преобладает над основным предметно-логическим значением атрибутивных компонентов, которое реализуется лишь частично. Компонент painfully в контексте высказывания реализует значение «causing emotional pain» (CALD) и отчасти значение «laborious, exacting, difficult» (MED); компонент clear метонимически реализует значение «free from doubt or confusion» (MED). Отношения контраста между этими компонентами реализуются на периферии их семантических значений по признакам «difficult, hard» - «effortless, easy».
Частотность использования оксюморонного ДЭ составляет 10 \% от общего числа ДЭ.

Другие виды семантических отношений между компонентами ДЭ реализуются редко и представлены отдельными примерами. В ходе исследования были обнаружены ДЭ, характеризующиеся отношениями неравенства: антиклимакс - immaculately clean Quaker bed (Beecher Stowe); тавтологическими отношениями - obscenely well obscenity (Hemingway); отношениями качества: антономасийный ДЭ - beastly English pride (Kipling2 , p. 192); отношениями количества: гипербола - microscopically small footboy (Dickens-2, p. 475), отношениями, реализующими иронию - handcuffs invitingly extended (Dickens-1, p. 30). ДЭ с перечисленными выше видами связи составляют $4 \%$ от общего количества проанализированных двухступенчатых эпитетов. Особенности грамматической структуры ДЭ не допускают таких видов семантической связи, как каламбур, хиазм или зевгма, поскольку они основаны на соотношении семантических и синтаксических средств и предполагают более свободную структурную организацию.

Принимая во внимание структурно-семантические особенности ДЭ, необходимо учесть, что границы между разными видами ДЭ нежесткие, иногда трудно устанавливаемые. Так, сложно разграничить синонимический и уточняющий ДЭ в таких выражениях, как, например, (1) tremulously uncertain hands (Wilde, с. 220) или (2) strictly restrained zeal (Bronte), в которых определяющие единицы могут как конкретизировать объект, так и реализовать отношения синонимии. Более того, ДЭ (1) с позиций парадигматики можно рассматривать как метонимический эпитет, а семантические отношения между атрибутивными компонентами и объектом в ДЭ (2) можно рассматривать как отношения контраста по признакам «passionless - passionate». В некоторых ДЭ за счет усилительного наречия может быть актуализирован элемент гиперболизации, например, в оксюморонном ДЭ amusements intolerably dull (Thackeray), метафорическом ДЭ water deadly still (Conrad) или синонимическом ДЭ dreadfully severe stare (Dickens-1, p. 141). 


\section{Выводы}

Итак, ДЭ представляет собой выразительное средство с заданной морфолого-синтаксической структурой, которая в значительной степени детерминирует его семантические характеристики и выразительный потенциал, обусловливая широкий спектр семантических связей между его компонентами. Предложенная в статье классификация семантических отношений между компонентами ДЭ отличается от общепринятых классификаций эпитетов и строится с учетом его двойственной лингвистической природы, а именно наличия у него, с одной стороны, грамматической структуры, определяющей его синтагматическую специфику, а с другой - широкого семантического потенциала. В связи с этим с опорой на классификацию стилистических ресурсов Ю.М. Скребнева семантические отношения между компонентами ДЭ рассмотрены в аспекте парадигматики (метафорический и метонимический ДЭ) и синтагматики (уточняющий, синонимический и оксюморонный и другие ДЭ). При этом граница между тем или иным типом семантической связи не является жесткой: один ДЭ может одновременно реализовать несколько видов семантических отношений. Помимо преобладающих типов семантической связи (взаимодополнительности, тождества и контраста) в структуре ДЭ могут быть реализованы тавтологические отношения, а также отношения неравенства (антиклимакс), качества (антономасия), количества (гипербола) и иронии. Однако данные виды семантической связи представлены единичными примерами.

\section{СПИСОК ЛИТЕРАТУРЫ}

Арнольд И. В., 1999. Семантика. Стилистика. Интертекстуальность : сб. ст. СПб. : Изд-во СПбГУ. $443 \mathrm{c}$.

Веселовский А. Н., 1989. Историческая поэтика. М. : Высшая школа. $408 \mathrm{c}$.

Гальперин И. Р., 1958. Очерки по стилистике английского языка. М. : Изд-во лит. на иностр. яз. $459 \mathrm{c}$.

Жирмунский В. М., 1992. К вопросу об эпитете // Памяти В.Н. Сакурина : сб. ст. М. : Наука. С. 52-60.

Сандакова М. В., 2006. Метонимия качественного прилагательного и его синтаксическая позиция // Актуальные проблемы лингвистики
XXI века : сб. тез. и ст. по материалам Междунар. науч. конф. (г. Киров, 6-7 дек. 2006 г.). Киров : Изд-во Вят. гос. гуманит. ун-та. С. 222-229.

Скребнев Ю. М., 2003. Основы стилистики английского языка. М. : Астрель, АСТ. 221 с.

Сусов И. П., 1970. О двух концепциях парадигматического анализа в синтаксисе // Синтагматика, парадигматика и их взаимоотношения на уровне синтаксиса : материалы науч. конф. Рига: [б. и.]. С. 169-173. URL: http://homepages. tversu.ru/ ips/IPS1970d.html (дата обращения: 14.09.2017).

Томашевский Б. В., 2010. Стилистика / отв. ред. А. Б. Муратов. М. : ЛИБРОКОМ. 282 с.

Турсунова Л. А., 1973. Структурные типы и стилистические функции эпитета в языке английской художественной литературы XX века : дис. ... канд. филол. наук. М. 198 с.

Corbett E. P. J., 1969. Rhetorical Analysis of Literary Works. London : Oxford University Press, Inc. $272 \mathrm{p}$.

Cruse D. A., 2011. Meaning in Language. An Introduction to Semantics and Pragmatics. Great Britain, Oxford : Oxford University Press. 131 p.

Galperin I. R., 1971. Stylistics. Moscow : Higher school. $343 \mathrm{p}$.

Kukharenco V.A., 1986. A Book of Practice in Stylistics. М. : Высшая школа. 144 c.

Murphy M. L., 2003. Semantic Relations and the Lexicon. UK, Cambridge : Cambridge University Press. 292 p.

Peters I., Weller K., 2008. Paradigmatic and Syntagmatic relations in Knowledge Organisation Systems // Information-Wissenschaft und Praxis. Vol. 59, № 2. P. 100-107. URL: https://pdfs. semanticscholar.org/e041/b7c5cca6968300c 1a96a8020aabdc54f4a47.pdf (date of access: 14.09.2017).

Skrebnev Yu. M., 1994. Fundamentals of English stylistics. Moscow : Editorial УРCC, 1994. $240 \mathrm{p}$.

\section{ИСТОЧНИКИ И СЛОВАРИ}

Beecher Stowe - Beecher Stowe H. Uncle Tom's Cabin. URL: http://booksonline.com.ua/view.php? book=160502 (date of access: 09.12.2016).

Bronte - Bronte Ch. Jane Eyre. URL: http://www. gutenberg.org/files/1260/1260-h/1260-h.htm (date of access: 09.12.2016).

$C A L D$ - Cambridge Advanced Learner's Dictionary. Cambridge : Cambridge University Press, 2005. $1584 \mathrm{p}$.

Collins - Collins W. The Woman in White. URL: http:// www.freeclassicebooks.com/Wilkie\%20Collins/ 
The $\% 20$ Woman $\% 20$ in $\% 20$ White.pdf (date of access: 24.02.2018).

Conrad - Conrad J. Lord Jim. URL: http://www. gutenberg.org/files/5658/5658-h/5658-h.htm (date of access: 09.12.2016).

Dickens-1 - Dickens Ch. Great Expectations. Penguin Books Ltd., 1994. 443 p.

Dickens-2 - Dickens Ch. Little Dorrit. Penguin Books Ltd., 1994. 848 p.

Fitzgerald-Fitzgerald F. S. The Beautiful and Damned. URL: https://fictionbook.ru/author/francis scott_fitzgerald/the_beautiful_and_damned/ read_online.html (date of access: 09.12.2016).

Fowles-1 - Fowles J. The Ebony Tower. M. : Прогресc, $1974.250 \mathrm{c}$.

Fowles-2-Fowles J. The Enigma. M. : Прогресс, 1974. $250 \mathrm{c}$.

Hemingway - Hemingway E. For Whom the Bell Tolls. URL: https://royallib.com/read/heminguey_ ernest/For_Whom_The_Bell_Tolls.html\#0 (date of access: 09.12.2016).

James - James H. The Portrait of a Lady. Wordsworth Classics, $1999.504 \mathrm{p}$.

Kipling-1 - Kipling R. With the Main Guard. Indian Tales. URL: http://www.gutenberg.org/dirs/ etext05/8indt10h.htm (date of access: 09.12.2016).

Kipling-2 - Kipling R. Kim. Wordsworth Classics, $1995.247 \mathrm{p}$.

Kipling-3 - Kipling R. The Light That Failed. M. : Прогресс, 1975. 285 c.

Lewis - Lewis M. The Monk. URL: https://royallib. com/read/Lewis_Matthew/the_monk.html\#0 (date of access: 09.12.2016).

Maugham - Maugham W. S. Cakes and Ale or the Skeleton in the Cupboard. M. : Менеджер, 2006. $256 \mathrm{c}$.

$M E D$ - Macmillan English Dictionary for Advanced Learners / ed. by M. Shovel. Oxford, 2007. 1854 p.

$O A L D$ - Oxford Advanced Learner's Dictionary / ed. McIntosh J. Turnbull. Oxford University Press, 2005. 1952 p.

Thackeray - Thackeray W. M. Vanity Fair. URL: http:/ /www.gutenberg. org/files/599/599-h/599-h.htm (date of access: 09.12.2016).

Updike - Updike J. Rabbit, Run. URL: https:// royallib.com/read/Updike_John/Rabbit_Run. html\#0 (date of access: 09.12.2016).

Wilde - Wilde O. The Picture of Dorian Gray // Избранные произведения : в 2 т. М. : Прогресс, 1979. T. $1.346 \mathrm{c}$.

\section{REFERENCES}

Arnold I.V., 1999. Semantics. Stylistics. Intertextuality. Saint Petersburg, Izd-vo St. Petersburg. un-ta. 443 p.
Veselovskiy A.N., 1958. Historical Poetics. Moscow, Vysshaya Shkola Publ. 408 p.

Galperin I.R., 1958. Essays on Stylistics of the English Language. Moscow, Prosveshchenie Publ. 459 p.

Zhirmunskiy V.M., 1992. On the Epithet. Sbornik statei pamyati V.N. Sakurina [Collected Papers in the Memory of V.N. Sakurin]. Moscow, NaukaPubl., pp. $52-60$

Sandakova M.V., 2006. Metonomy of the Qualitative Adjective and Its Syntactic Position. Aktualnye problem lingvistiki XXI veka: sb. tez. $i$ st. [Essential Problems of Linguistics of the $21^{\text {st }}$ Century: Collected Theses and Papers]. Kirov, Izd-vo VGGU, pp. 222-229.

Skrebnev Yu.M., 2003. Fundamentals of Stylistics of the English Language. Moscow, Astrel; AST Publ. 221 p.

Susov I.P., 1970. On Two Conceptions of Paradigmatic Analysis in Syntax. Sintagmatika, paradigmatika i ikh vzaimootnosheniya na urovne sintaksisa: materialy nauch. konf. [Sintagmatics, Paradigmatics and Their Interrelations at the Syntactic Level. Proceedings of Academic Conference]. Riga, pp. 169-173. URL: http:// homepages.tversu.ru/ ips/IPS1970d.html (accessed September 14, 2017).

Tomashevskiy B.V., 2010. Stylistics. Moscow, LIBROCOM Publ. 282 p.

Tursunova L.A., 1973. Structural Types and Stylistic Functions of Epithet in the Language of Fiction of the $20^{\text {th }}$ Century. Dissertation for Candidate of Sciences in Philology Degree. Moscow. 198 p.

Corbett E.P.J., 1969. Rhetorical Analysis of Literary Works. London, Oxford University Press, Inc. $272 \mathrm{p}$.

Cruse D.A., 2011. Meaning in Language. An Introduction to Semantics and Pragmatics. Oxford, Oxford University Press. 131 p.

Galperin I.R., 1971. Stylistics. Moscow, Vysshaya shkola Publ. 343 p.

Kukharenko V.A., 1986. A Book of Practice in Stylistics. Moscow, Vysshaya shkola Publ. $144 \mathrm{p}$.

Murphy M.L., 2003. Semantic Relations and the Lexicon. Cambridge, Cambridge University Press. 292 p.

Peters I., Weller K., 2008. Paradigmatic and Syntagmatic relations in Knowledge Organisation Systems. Information-Wissenschaft und Praxis, vol. 59, no. 2, pp. 100-107. URL: https://pdfs. semanticscholar.org/e041/b7c5cca6968300 c1a96a8020aabdc54f4a47.pdf (accessed September 14, 2017).

Skrebnev Yu.M., 1994. Fundamentals of English stylistics. Moscow, Editorial URSS Publ. 240 p. 
О.Л. Любовская. Классификация семантических отношений компонентов двухступенчатого эпитета

\section{SOURCES AND DICTIONARIES}

Beecher Stowe H. Uncle Tom's Cabin. URL: http:// booksonline.com.ua/view.php?book=160502 (accessed December 9, 2016).

Bronte Ch. Jane Eyre. URL: http://www.gutenberg.org/ files/1260/1260-h/1260-h.htm (accessed December 9, 2016).

Cambridge Advanced Learner's Dictionary. Cambridge University Press, 2005. 1584 p.

Collins W. The Woman in White. URL: http://www. freeclassicebooks.com/Wilkie\%20Collins/ The\%20Woman\%20in\%20White.pdf(accessed February 24, 2018).

Conrad J. Lord Jim. URL: http://www.gutenberg.org/ files/5658/5658-h/5658-h.htm (accessed December 9, 2016).

Dickens Ch. Great Expectations. Penguin Books, $1994.443 \mathrm{p}$.

Dickens Ch. Little Dorrit. Penguin Books Ltd., 1994. $848 \mathrm{p}$.

Fitzgerald F.S. The Beautiful and Damned. URL: https:// fictionbook.ru/author/francis_scott_fitzgerald/ the beautiful and_damned/read_online.html (accessed December 9, 2016).

Fowles J. The Ebony Tower. Moscow, Progress Publ., $1974.250 \mathrm{p}$.

Fowles J. The Enigma. Moscow, Progress Publ., 1974. $250 \mathrm{p}$.
Hemingway E. For Whom the Bell Tolls. URL: https:// royallib.com/read/heminguey ernest/For Whom The Bell Tolls.html\#0 (accessed December 9, 2016).

James H. The Portrait of a Lady. Wordsworth Classics, $1999.504 \mathrm{p}$.

Kipling R. With the Main Guard. Indian Tales. URL: http://www.gutenberg.org/dirs/etext05/8indt10h. htm (accessed December 9, 2016).

Kipling R. Kim. Wordsworth Classics, 1995. 247 p.

Kipling R. The Light That Failed. Moscow, Progress Publ., 1975. $285 \mathrm{p}$.

Lewis M. The Monk. URL: https://royallib.com/read/ Lewis Matthew/the monk.html\#0 (accessed December 9, 2016).

Maugham W.S. Cakes and Ale or the Skeleton in the Cupboard. Moscow, Manager Publ., 2006. 256 p.

Shovel M., ed. Macmillan English Dictionary for Advanced Learners. Oxford, 2007. $1854 \mathrm{p}$.

McIntosh J.T., ed. Oxford Advanced Learner's Dictionary. Oxford University Press, 2005. 1952 p.

Thackeray W.M. Vanity Fair. URL: http://www. gutenberg.org/files/599/599-h/599-h.htm (accessed December 9, 2016).

Updike J. Rabbit, Run. URL: https://royallib.com/read/ Updike_John/Rabbit_Run.html\#0 (accessed December 9, 2016).

Wilde O. The Picture of Dorian Gray. O. Wilde. Selected works. In 2 vols. Moscow, Progress Publ., 1979, vol. 1.346 p.

\section{Information about the Author}

Olga L. Lyubovskaya, Candidate of Sciences (Philology), Assistant, Department of the English Language for the Faculty of Arts, Saint Petersburg State University, Universitetskaya Emb., 7-9, 199034 Saint Petersburg, Russia, spbu@spbu.ru, https://orcid.org/0000-0002-9476-3644

\section{Информация об авторе}

Ольга Леонидовна Любовская, кандидат филологических наук, ассистент кафедры английского языка для факультета искусств, Санкт-Петербургский государственный университет, Университетская наб., 7-9, 199034 г. Санкт-Петербург, Россия, spbu@spbu.ru, https://orcid.org/00000002-9476-3644 